\title{
Vitamin $C$ for $\geq 5$ days is associated with decreased hospital mortality in sepsis subgroups: a nationwide cohort study
}

\author{
Sun-Young Jung ${ }^{1,2 \dagger}$, Min-Taek Lee ${ }^{2 \dagger}$, Moon Seong Baek ${ }^{3}$ and Won-Young Kim ${ }^{3^{*}}$ (i)
}

\begin{abstract}
Background: Previous randomized trials of vitamin C, hydrocortisone, and thiamine on sepsis were limited by shortterm vitamin C administration, heterogeneous populations, and the failure to evaluate each component's effect. The purpose of this study was to determine whether vitamin $C$ alone for $\geq 5$ days or in combination with corticosteroids and/or thiamine was associated with decreased mortality across the sepsis population and subpopulation.

Methods: Nationwide population-based study conducted using the Korean National Health Insurance Service database. A total of 384,282 adult patients with sepsis who were admitted to the intensive care unit were enrolled from January 2017 to December 2019. The primary outcome was hospital mortality, while the key secondary outcome was 90-day mortality.

Results: The mean [standard deviation] age was 69.0 [15.4] years; 57\% were male; and 36,327 (9\%) and 347,955 did and did not receive vitamin C, respectively. After propensity score matching, each group involved 36,327 patients. The hospital mortality was lower by $-0.9 \%$ in the treatment group $(17.1 \%$ vs $18.0 \%$; $95 \%$ confidence interval, -1.3 to $-0.5 \% ; p<0.001)$, a significant but extremely small difference. However, mortality decreased greater in patients who received vitamin $C$ for $\geq 5$ days (vs $1-2$ or $3-4$ days) (15.8\% vs $18.8 \%$ vs $18.3 \% ; p<0.001)$. Further, vitamin $C$ was associated with a lower hospital mortality in patients with older age, multiple comorbidities, pneumonia, genitourinary infection, septic shock, and mechanical ventilation. Consistent findings were found for 90-day mortality. Moreover, vitamin $C$ alone or in combination with thiamine was significantly associated with decreased hospital mortality.

Conclusions: Intravenous vitamin $\mathrm{C}$ of $\geq 5$ days was significantly associated with decreased hospital and 90-day mortality in sepsis patients. Vitamin C combined with corticosteroids and/or thiamine in specific sepsis subgroups warrants further study.
\end{abstract}

Keywords: Ascorbic acid, Mortality, Sepsis, Septic shock, Steroids, Thiamine

*Correspondence: wykim81@cau.ac.kr

†Sun-Young Jung and Min-Taek Lee contributed equally to this manuscript

${ }^{3}$ Division of Pulmonary and Critical Care Medicine, Department of Internal Medicine, Chung-Ang University Hospital, Chung-Ang University College of Medicine, Seoul, Republic of Korea

Full list of author information is available at the end of the article

\section{Background}

Sepsis is a life-threating organ dysfunction caused by a dysregulated host response to infection [1]. Importantly, sepsis results in more than 5 million deaths globally each year, thus making it a major public health concern [2]. Accordingly, novel therapeutic interventions for sepsis have been explored over the last several decades. However, these have demonstrated inconsistent benefits [3].

Vitamin $\mathrm{C}$ downregulates proinflammatory cytokines; maintains the endothelial barrier; and facilitates the original author(s) and the source, provide a link to the Creative Commons licence, and indicate if changes were made. The images or other third party material in this article are included in the article's Creative Commons licence, unless indicated otherwise in a credit line to the material. If material is not included in the article's Creative Commons licence and your intended use is not permitted by statutory regulation or exceeds the permitted use, you will need to obtain permission directly from the copyright holder. To view a copy of this licence, visit http://creativecommons.org/licenses/by/4.0/. The Creative Commons Public Domain Dedication waiver (http://creativecommons.org/publicdomain/zero/1.0/) applies to the data made available in this article, unless otherwise stated in a credit line to the data. 
production of catecholamines, vasopressin, and cortisol $[4,5]$. A recent retrospective study showed that the combination of vitamin $\mathrm{C}$, hydrocortisone, and thiamine has a substantial survival benefit in patients with sepsis [6]. As such, numerous randomized trials of vitamin $\mathrm{C}$ alone or in combination with hydrocortisone and thiamine for sepsis have been conducted, although neither treatment was associated with significantly improved outcomes [7-10]. However, these trials had insufficient power for detecting differences in mortality and limited the use of vitamin $C$ to a maximum of 4 days. Low-dose corticosteroids have been shown to reverse septic shock [11, 12], although the use of hydrocortisone was not controlled in most of these trials $[7,9,10]$. A recent network metaanalysis evaluated larger samples to estimate the effect of vitamin $\mathrm{C}$, corticosteroids, and thiamine alone or in combination on mortality and other outcomes [13]. However, this analysis did not assess the treatment duration and may be limited by heterogeneity of the published studies.

To address current knowledge gaps, this study aimed to investigate whether vitamin $C$ for $\geq 5$ days was associated with improved hospital mortality across populations with sepsis. Toward this goal, data using the Korean National Health Insurance Service (NHIS) database were analyzed. Various subgroup analyses were also performed to analyze mortality differences according to sepsis subpopulation and combination with corticosteroids and/or thiamine.

\section{Methods}

\section{Data source and study population}

This retrospective cohort study collected data from the NHIS database, which consists of reimbursement claims from all types of healthcare facilities among over $97 \%$ of the population in Korea (51.1 million in 2018) [14]. The database contains inpatient information including demographics; primary and secondary diagnoses; admission history; hospital length of stay; discharge status; date of death (if applicable); diagnoses; and procedure and prescription charges. The reimbursement plan is based on fee-for-service and does not incentivize upcoding as reimbursement is not related to a specific diagnosis. Diagnoses were coded based on the International Classification of Diseases, 10th Revision (ICD-10), exclusively by attending physicians. There are no governmental mandates for sepsis care and reporting in Korea.

In this study, adult (age $\geq 18$ years) patients with sepsis admitted to the intensive care unit (ICU) between January 2017 and December 2019 were evaluated. Sepsis was defined following the Sepsis-3 definition [1] as infection and organ dysfunction before hospital discharge, and the patients were identified using the ICD-10 codes for infection (Additional file 1: Appendix 1) and organ dysfunction (Additional file 2: Table S1). The presence of septic shock was determined according to the relevant ICD-10 code (R57.2) or the administration of vasopressors such as norepinephrine, epinephrine, vasopressin, and dopamine. According to the Sepsis-3 criteria [1], vasopressor use equates to a score greater than or equal to 2 in the Sequential Organ Failure Assessment (SOFA) cardiovascular subscore and was used as a reasonable surrogate of sepsis severity. The exclusion criteria were age $<18$ years; pregnancy or related condition; palliative care; cardiac arrest; and multiple ICU admissions. In the NHIS database, it is not feasible to temporally associate diagnoses (sepsis) with prescription charges (vitamin C) during hospitalization due to lack of time stamps, and immortal time bias may be a significant concern. To minimize bias, two analytical approaches were employed, namely, (1) an analysis excluding patients who died or were discharged within 2 days of ICU admission to prevent patients who died early in their hospital course from introducing bias and (2) an analysis assessing outcomes with stratification according to the follow-up period given the study intervention is not temporally defined [15].

The need for ethical approval was waived by the Institutional Review Board of Chung-Ang University owing to the de-identified patient data (1041078-202001-HR012-01). This study complied with the Strengthening the Reporting of Observational Studies in Epidemiology guidelines.

\section{Vitamin C, corticosteroids, and thiamine}

Patients who did and did not receive at least one charge for high-dose intravenous (IV) vitamin $\mathrm{C}$ during the index hospitalization were categorized into the treatment and control groups, respectively. Corticosteroid or thiamine use was defined as at least one charge for IV corticosteroids or IV thiamine during the index hospitalization, respectively. The daily dose of each drug was not calculated because drug prescriptions do not necessarily reflect actual treatment. However, the dosing of vitamin $\mathrm{C}$ may have been quite consistent around $6 \mathrm{~g} /$ day based on previous studies conducted in Korea [16, 17]. Total duration of use was determined for all three drugs. The detailed codes for IV vitamin C, corticosteroids, and thiamine are shown in Additional file 2: Table S2.

\section{Data collection, definitions, and outcomes}

Baseline patient characteristics included age; sex; Charlson comorbidities [18] defined using claim records within 1 year prior to admission; immunosuppression (malignancy, human immunodeficiency virus infection, organ transplant, or immunosuppressive therapy); previous steroid use (oral or IV for $\geq 30$ days during the preceding 
year); emergency department presentation; and admitting department. The sites of infection were identified using ICD-10 codes (Additional file 2: Table S3), and hospital capacity was measured by the number of beds. Procedure codes were used to extract clinical care variables such as conventional oxygen therapy, high-flow nasal cannula, mechanical ventilation, and renal replacement therapy.

The primary outcome was hospital mortality. The secondary outcomes were 90-day mortality, vasopressor days, ventilator days, ICU and hospital lengths of stay, and hospital costs.

\section{Statistical analysis}

Continuous data were reported as the mean (standard deviation, SD) and were compared using Student's $t$-test or Kruskal-Wallis test, as appropriate. Categorical data were reported as the number (percentage) and were compared using chi-square test or Fisher's exact test, as appropriate. Outcome data were presented as the mean of all paired differences with 95\% confidence intervals (CIs). Propensity score (PS) analysis was performed to create a matched cohort of patients who had differences in the administration of vitamin $C$ but were similar in other measured variables [19]. The conditional probability of patients to receive vitamin $\mathrm{C}$ given the individual covariates is unclear. Thus, PS in each patient was estimated using logistic regression model that included all available baseline covariates (Table 1), except for thiamine use. Patients in the treatment and control groups were matched 1:1 without replacement, using nearest neighbor matching based on a greedy matching algorithm [20]. To ensure good matches, a caliper of 0.2 of the SD of the logit of the PS was defined. Standardized mean differences were calculated among covariates before and after matching, and a difference of $\leq 0.10$ was considered well-balanced. Survival to 90 days was plotted using Kaplan-Meier curves and compared between the PS-matched treatment and control groups using log-rank tests. Logistic regression analysis was performed to identify the risk factors for hospital mortality.

The duration-response relationship between vitamin C and clinical outcomes was assessed by comparing vitamin C for 1-2 days, $3-4$ days, and $\geq 5$ days. The cut-off values were based on a recent meta-analysis [17]. The duration of $\geq 5$ days was selected based on a hypothesis that limiting the use of vitamin $C$ to 4 days, which was assessed in most previous studies, may not translate to a survival benefit. Subgroup analyses for the primary and secondary outcomes were performed on subpopulations determined a priori from baseline covariates associated with hospital mortality. These included age $\geq 70$ or $<70$ years; male or female; Charlson Comorbidity Index $(\mathrm{CCI}) \geq 3$ or $<3$; pneumonia; gastrointestinal infection; genitourinary infection; septic shock; mechanical ventilation; and renal replacement therapy. Pneumonia was selected on the basis that vitamin $C$ depletion and elevated oxidative stress were observed in patients with pneumonia sepsis [21], and vitamin $C$ administration may decrease oxidative stress and improve survival. Moreover, vitamin $\mathrm{C}$ may reduce genitourinary infection by production of reactive nitrogen species and inhibition of biofilm formation on the urinary catheters [22, 23]. Two sensitivity analyses were performed, namely, (1) an analysis including only patients who survived to hospital discharge and (2) an analysis between patients who received vitamin $\mathrm{C}$ for $\geq 5$ days and matched controls. Subgroup analyses including vitamin $C$ only versus various combinations with corticosteroids and/or thiamine with stratification according to the duration of use ( $<5$ days vs $\geq 5$ days) were also performed. The PS-matched odds ratios (ORs) and $95 \%$ CIs for hospital mortality were calculated and shown using forest plots.

There were no missing values in the dataset. No adjustment was made for multiple comparisons, and the findings of secondary analyses should be considered hypothesis generating. All analyses were performed using SAS software (version 9.4; SAS Institute, Cary, NC, USA). A two-tailed $p$ value of $<0.05$ was considered statistically significant.

\section{Results \\ Patient characteristics}

Among the 886,553 eligible patients, 502,271 were excluded (Fig. 1). Thus, 384,282 patients were included in the analysis. Of them, 36,327 (9\%) and 347,955 patients were categorized to the treatment and control groups, respectively. The baseline characteristics of the unmatched patients are shown in Additional file 2: Table S4. The mean (SD) age was 69.0 (15.4) years, and $57 \%$ were male. The treatment group were more likely to be older, have a higher CCI, have pneumonia and genitourinary infection, and receive thiamine. Meanwhile, the control group were more likely to be admitted in a higher volume hospital, have septic shock, and require mechanical ventilation.

\section{Propensity score analysis}

After matching, each group involved 36,327 patients. The baseline characteristics were generally well-matched between the two groups (Table 1). The major sites of infection were the lungs, gastrointestinal tract, and genitourinary tract, and the most common type of organ dysfunction was respiratory failure. Almost half of the patients had septic shock. There was no significant between-group difference in the use of corticosteroids. 
Table 1 Baseline patient characteristics in the matched cohort

\begin{tabular}{|c|c|c|c|}
\hline Characteristics & Vitamin C $(n=36,327)$ & Control $(n=36,327)$ & SMD \\
\hline Age, mean (SD), years & $70.7(15.2)$ & $70.7(15.5)$ & 0.03 \\
\hline Sex, No. (\%) & & & -0.02 \\
\hline Male & $20,093(55)$ & $19,919(55)$ & \\
\hline Female & $16,234(45)$ & $16,408(45)$ & \\
\hline \multicolumn{4}{|l|}{ Comorbidities, No. (\%) } \\
\hline Diabetes & $15,369(42)$ & $15,024(41)$ & 0.02 \\
\hline Hypertension & $24,236(67)$ & $23,996(66)$ & 0.01 \\
\hline Myocardial infarction & $1224(3)$ & $1109(3)$ & 0.02 \\
\hline Congestive heart failure & $6154(17)$ & $5795(16)$ & 0.03 \\
\hline Cerebrovascular disease & $9683(27)$ & $9483(26)$ & 0.01 \\
\hline Chronic pulmonary disease & $16,133(44)$ & $15,852(44)$ & 0.02 \\
\hline Chronic liver disease & $11,268(31)$ & $11,132(31)$ & 0.008 \\
\hline Chronic kidney disease & $3929(11)$ & $3651(10)$ & 0.03 \\
\hline Malignancy & $6965(19)$ & $6679(18)$ & 0.02 \\
\hline Charlson Comorbidity Index, mean (SD) & $3.0(1.9)$ & $2.9(1.9)$ & 0.05 \\
\hline Immunosuppression, No. (\%) ${ }^{\mathrm{a}}$ & $10,313(28)$ & $9860(27)$ & 0.03 \\
\hline Previous steroid use, No. $(\%)^{b}$ & $4461(12)$ & $4216(12)$ & 0.02 \\
\hline \multicolumn{4}{|l|}{ Site of infection, No. (\%) } \\
\hline Lung & $11,088(31)$ & $10,919(30)$ & 0.01 \\
\hline Gastrointestinal tract & $4801(13)$ & $4570(13)$ & 0.02 \\
\hline Genitourinary tract & $4644(13)$ & $4518(12)$ & 0.01 \\
\hline Hospital capacity, No. (\%) & & & 0.04 \\
\hline$<500$ beds & $18,373(51)$ & $18,477(51)$ & \\
\hline $500-1000$ beds & $14,259(39)$ & $14,546(40)$ & \\
\hline$\geq 1000$ beds & $3695(10)$ & $3304(9)$ & \\
\hline Presented to emergency department, No. (\%) & $28,337(78)$ & $28,602(79)$ & -0.02 \\
\hline Medical department, No. (\%) & $25,723(71)$ & $25,455(70)$ & 0.02 \\
\hline Septic shock, No. (\%) & $18,077(50)$ & $17,569(48)$ & 0.03 \\
\hline \multicolumn{4}{|l|}{ Organ dysfunction, No. (\%) } \\
\hline Cardiovascular & $18,325(50)$ & $17,793(49)$ & 0.03 \\
\hline Respiratory & $33,080(91)$ & $33,133(91)$ & -0.005 \\
\hline Neurologic & $2008(6)$ & $1848(5)$ & 0.02 \\
\hline Hematologic & $1032(3)$ & $958(3)$ & 0.01 \\
\hline Hepatic & $621(2)$ & $634(2)$ & -0.003 \\
\hline Renal & $4801(13)$ & $4453(12)$ & 0.03 \\
\hline Metabolic & $470(1)$ & $481(1)$ & -0.003 \\
\hline No. of organ dysfunctions, No. (\%) & & & 0.05 \\
\hline 1 & $14,955(41)$ & $15,697(43)$ & \\
\hline 2 & $14,870(41)$ & $14,602(40)$ & \\
\hline 3 & $4290(12)$ & $3940(11)$ & \\
\hline$\geq 4$ & $706(2)$ & $702(2)$ & \\
\hline Corticosteroids, No. (\%) & $13,968(38)$ & $13,759(38)$ & 0.01 \\
\hline Thiamine, No. (\%) & $7745(21)$ & $3517(10)$ & 0.33 \\
\hline Conventional oxygen therapy, №. (\%) & $31,670(87)$ & $31,858(88)$ & -0.02 \\
\hline High-flow nasal cannula, No. (\%) & $4059(11)$ & $3862(11)$ & 0.02 \\
\hline Mechanical ventilation, №. (\%) & $10,793(30)$ & $10,404(29)$ & 0.02 \\
\hline Renal replacement therapy, No. (\%) & $2628(7)$ & $2494(7)$ & 0.01 \\
\hline
\end{tabular}

SMD standardized mean difference

${ }^{a}$ Immunosuppression included malignancy, human immunodeficiency virus infection, organ transplant, or immunosuppressive therapy

${ }^{b}$ Defined by oral or intravenous for $\geq 30$ days during the preceding year 


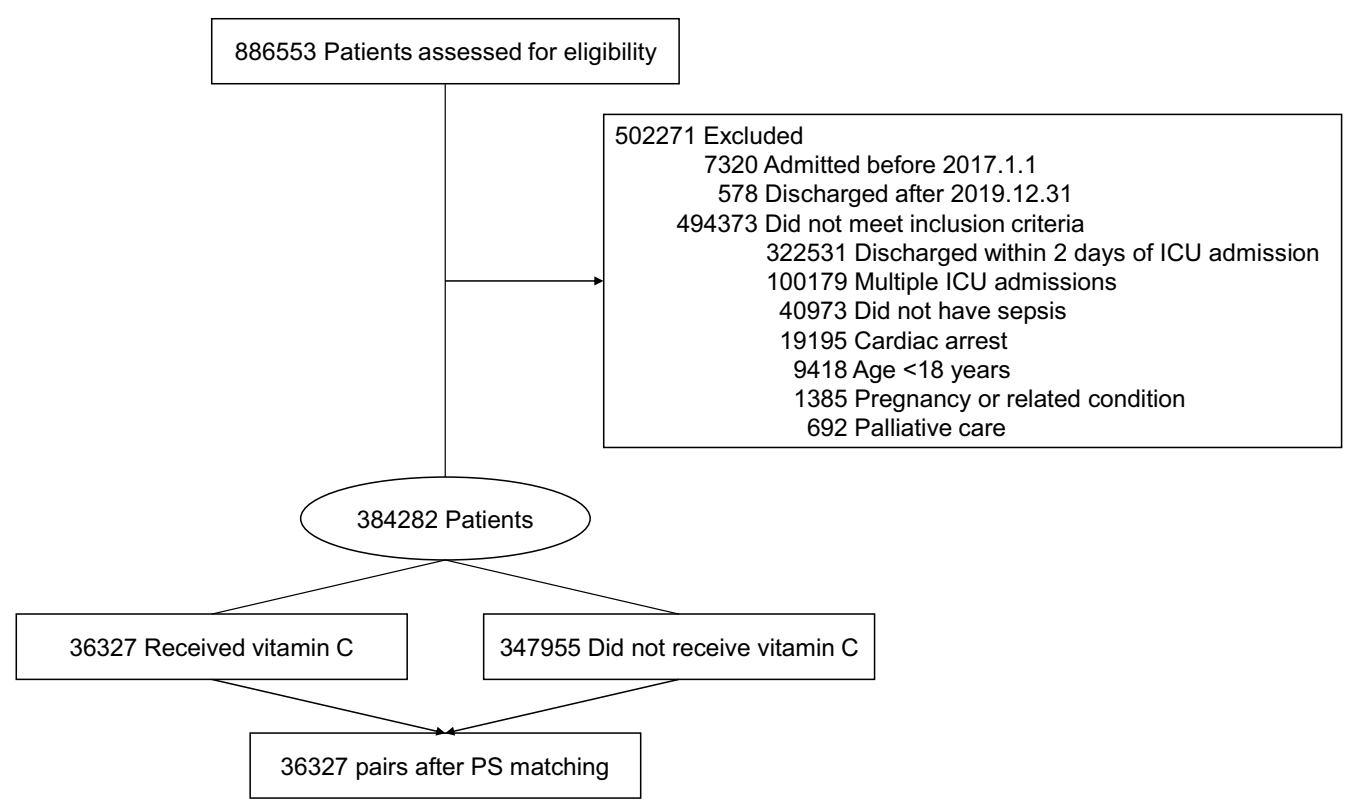

Fig. 1 Flow of patients in the nationwide cohort study for vitamin C in sepsis. ICU intensive care unit, PS propensity score

Meanwhile, there were significant differences in hospital mortality $(17.1 \%$ vs $18.0 \%$; difference, $-0.9 \%$; $95 \%$ CI -1.3 to $-0.5 \% ; p<0.001)$ and 90 -day mortality $(25.4 \%$ vs $27.0 \%$; difference, $-3.2 \%$; $95 \%$ CI -3.8 to $-2.6 \%$; $p<0.001$ ) (Table 2). These observations were not influenced when the patients were stratified according to the length of ICU stay ( $\leq 5$ days vs $>5$ days) (Additional file 2 : Table S5).

The survival curves were also significantly different between the two groups on log-rank test $(p<0.001)$ (Fig. 2a). Vasopressor days, ventilator days, ICU and hospital lengths of stay, and hospital costs were significantly higher in the treatment group (Table 2). Sensitivity analysis of patients who survived to hospital discharge demonstrated similar findings (Additional file 2: Table S6). Vitamin C use was associated with a significantly lower risk of hospital mortality (PSmatched OR, 0.94; 95\% CI 0.90-0.97) (Additional file 2: Table S7). Conversely, the use of corticosteroids was associated with a significantly higher risk of mortality (PS-matched OR, 1.94; 95\% CI 1.82-2.07).

Table 2 Primary and secondary outcomes

\begin{tabular}{|c|c|c|c|c|}
\hline Outcomes & Vitamin C $(n=36,327)$ & Control $(n=36,327)$ & Difference $(95 \% \mathrm{Cl})$ & $p$ value \\
\hline \multicolumn{5}{|l|}{ Primary outcome } \\
\hline Hospital mortality, No. (\%) & $6209(17.1)$ & $6538(18.0)$ & $-0.9(-1.3 \text { to }-0.5)^{\mathrm{a}}$ & $<0.001$ \\
\hline \multicolumn{5}{|l|}{ Secondary outcomes } \\
\hline 90-day mortality, No. (\%) & $9226(25.4)$ & $9820(27.0)$ & $-3.2(-3.8$ to -2.6$)$ & $<0.001$ \\
\hline Vasopressor days, mean (SD) & $\begin{array}{l}2.9(2.3) \\
{[n=17923]}\end{array}$ & $\begin{array}{l}2.7(2.0) \\
{[n=17441]}\end{array}$ & 0.12 (0.1 to 0.2$)$ & 0.002 \\
\hline Ventilator days, mean (SD) & $\begin{array}{l}9.5(18.8) \\
{[n=10793]}\end{array}$ & $\begin{array}{l}8.2(15.7) \\
{[n=10404]}\end{array}$ & $1.4(1.3$ to 1.5$)$ & $<0.001$ \\
\hline \multicolumn{5}{|l|}{ Length of stay, mean (SD), days } \\
\hline ICU & $10.6(17.5)$ & $8.6(14.2)$ & $2.02(2.0$ to 2.1$)$ & $<0.001$ \\
\hline Hospital & $27.1(41.6)$ & $21.0(22.1)$ & $6.0(5.9$ to 6.1$)$ & $<0.001$ \\
\hline Hospital costs, mean (SD), U.S. \$1000 & $13.3(19.0)$ & $12.1(25.2)$ & 1.19 (1.18 to 1.2$)$ & $<0.001$ \\
\hline
\end{tabular}

ICU intensive care unit

a The mean of all paired differences in the treatment group minus the control group 


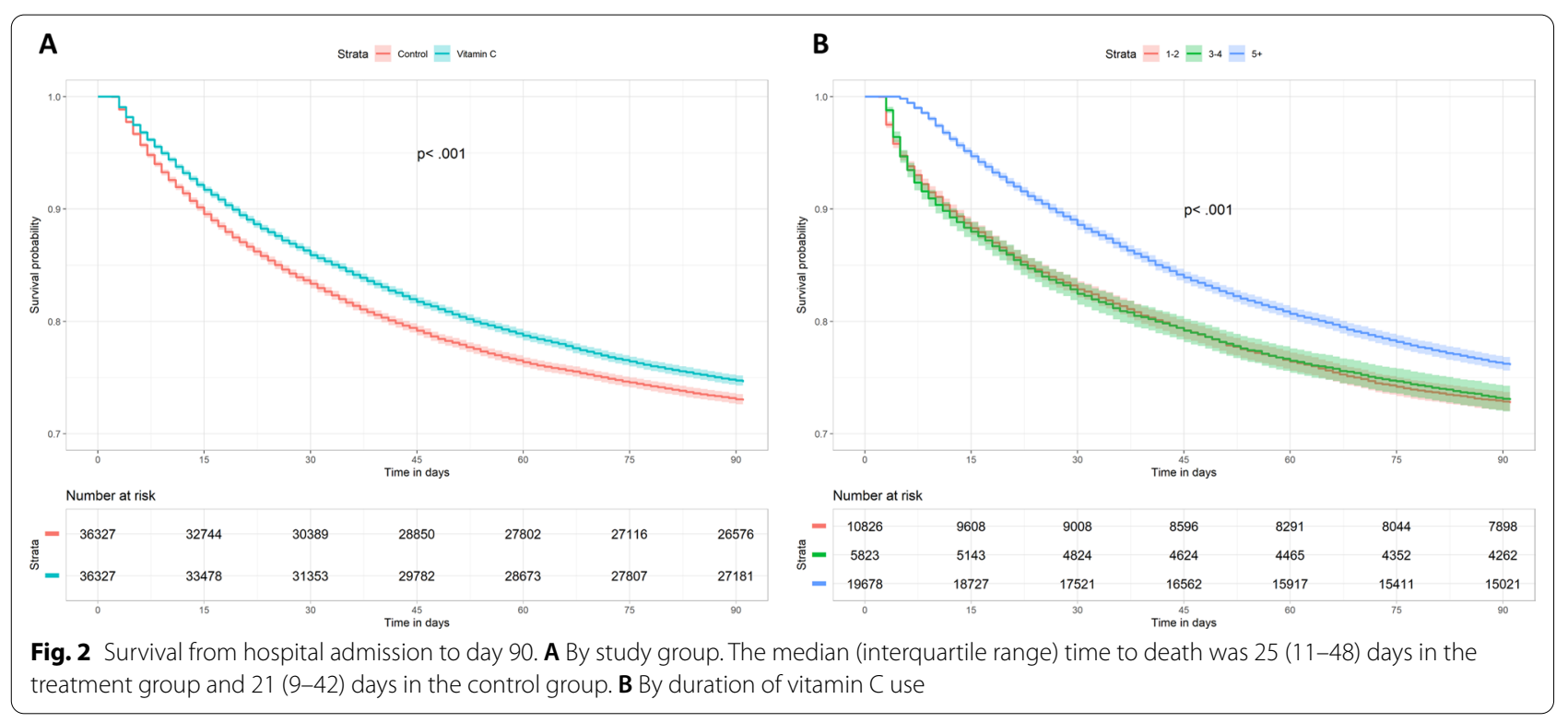

\section{Vitamin C duration and mortality}

Of the 36,327 patients in the treatment group, 10,826 (30\%) patients received vitamin $\mathrm{C}$ for $1-2$ days; 5823 (16\%) patients, 3-4 days; and 19,678 (54\%) patients, $\geq 5$ days. Among patients who received vitamin $\mathrm{C}$ for $\geq 5$ days, the median (interquartile range) duration of use was 11 ( 7 to 17) days. Compared with patients who received vitamin $C$ for $1-2$ or $3-4$ days, those treated for $\geq 5$ days showed significantly lower hospital $(18.8 \%$ vs $18.3 \%$ vs $15.8 \% ; p<0.001)$ and 90 -day mortality $(27.3 \%$ vs $27.0 \%$ vs $23.9 \% ; p<0.001$ ) rates (Additional file 2 : Table S8 and Fig. 2b). Patients who received vitamin C for $\geq 5$ days had significantly lower hospital and 90-day mortality rates compared to PS-matched controls (Additional file 2: Table S9). These findings were not influenced when the patients were stratified according to the length of ICU stay ( $\leq 5$ days vs $>5$ days) (Additional file 2 : Table S10). Vasopressor days, ventilator days, and ICU and hospital lengths of stay were also significantly higher in these patients (Additional file 2: Table S8).

\section{Subgroup analyses}

The primary and secondary outcomes among different subpopulations with sepsis are shown in Additional file 2: Table S11. Vitamin C use was associated with significantly lower risks of hospital and 90-day mortalities in patients with older age ( $\geq 70$ years), a higher number of comorbidities $(\mathrm{CCI} \geq 3)$, pneumonia, genitourinary infection, septic shock, and mechanical ventilation. Conversely, mortality did not differ according to gastrointestinal infection and renal replacement therapy between the treatment and control groups. There were also no sex differences in mortality, but it was significantly lower for patients who received vitamin C. In general, patients who received vitamin $\mathrm{C}$ had significantly higher vasopressor days, ventilator days, ICU and hospital lengths of stay, and hospital costs across all subgroups. The results of sensitivity analyses of patients who received vitamin $C$ for $\geq 5$ days were similar to the primary analyses, although mortality among patients with renal replacement therapy was significantly lower when vitamin $C$ was administered (Additional file 2: Table S12). The differential survival probabilities by subgroups are demonstrated in Additional file 3: Figs. S1 and S2.

\section{Vitamin C only vs combination therapy}

Figure 3 shows the results of subgroup analyses regarding the association between vitamin $C$ only and hospital mortality compared with a combined therapy with corticosteroids and/or thiamine. Forest plots for combinations demonstrated that vitamin $\mathrm{C}$ alone (any duration) or the combination of vitamin $\mathrm{C}$ (any duration) plus thiamine (any duration) was significantly associated with decreased mortality. However, any combination of vitamin $\mathrm{C}$ and corticosteroids, regardless of thiamine use, was significantly associated with increased mortality. The results of the primary analyses were generally consistent across all subpopulations (Additional file 3: Fig. S3), although the combination of vitamin $\mathrm{C}$ (any duration) plus corticosteroids $<5$ days was significantly associated with decreased mortality among patients with septic shock and mechanical ventilation. 
No. of deaths/patients $(\%)$

PS-matched OR $(95 \% \mathrm{CI})$

\begin{tabular}{lc}
\hline Vitamin C duration & \\
No Vitamin C & $6538 / 36327(18.0)$ \\
Vitamin C $<5 \mathrm{~d}$ & $3100 / 16649(18.6)$ \\
Vitamin C $\geq 5 \mathrm{~d}$ & $3109 / 19678(15.8)$ \\
Steroid duration & \\
No Vitamin C & $6538 / 36327(18.0)$ \\
Vitamin C (any duration) + no steroid & $2976 / 22359(13.3)$ \\
Vitamin C (any duration) + steroid $<5 \mathrm{~d}$ & $2183 / 11124(19.6)$ \\
Vitamin C (any duration) + steroid $\geq 5 \mathrm{~d}$ & $1050 / 2844(36.9)$ \\
Vitamin C $\geq 5 \mathrm{~d}$ & \\
No Vitamin C & $6538 / 36327(18.0)$ \\
Vitamin C $<5 \mathrm{~d}$ & $3100 / 16649(18.6)$ \\
Vitamin C $\geq 5 \mathrm{~d}+$ no thiamine + no steroid & $1298 / 10291(12.6)$ \\
Vitamin C $\geq 5 \mathrm{~d}+$ no thiamine + steroid $<5 \mathrm{~d}$ & $783 / 4791(16.3)$ \\
Vitamin C $\geq 5 \mathrm{~d}+$ no thiamine + steroid $\geq 5 \mathrm{~d}$ & $393 / 1191(33.0)$ \\
Vitamin C $\geq 5 \mathrm{~d}+$ thiamine $<5 \mathrm{~d}+$ no steroid & $48 / 489(9.8)$ \\
Vitamin C $\geq 5 \mathrm{~d}+$ thiamine $<5 \mathrm{~d}+$ steroid $<5 \mathrm{~d}$ & $48 / 235(20.4)$ \\
Vitamin C $\geq 5 \mathrm{~d}+$ thiamine $<5 \mathrm{~d}+$ steroid $\geq 5 \mathrm{~d}$ & $32 / 77(41.6)$ \\
Vitamin C $\geq 5 \mathrm{~d}+$ thiamine $\geq 5 \mathrm{~d}+$ no steroid & $185 / 1628(11.4)$ \\
Vitamin C $\geq 5 \mathrm{~d}+$ thiamine $\geq 5 \mathrm{~d}+$ steroid $<5 \mathrm{~d}$ & $179 / 643(27.8)$ \\
Vitamin C $\geq 5 \mathrm{~d}$ + thiamine $\geq 5 \mathrm{~d}+$ steroid $\geq 5 \mathrm{~d}$ & $143 / 333(42.9)$
\end{tabular}

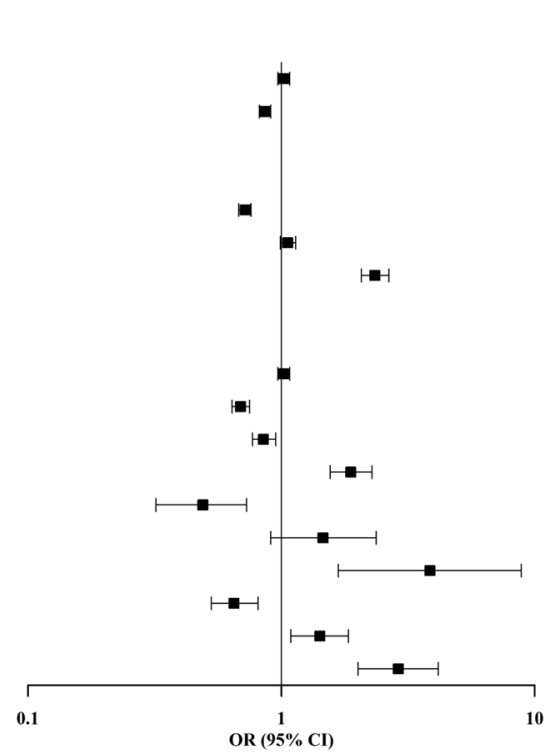

ref

$1.02(0.97-1.08)$

$0.86(0.82-0.91)$

ref

$0.72(0.68-0.76)$

$1.06(0.99-1.14)$

$2.34(2.07-2.66)$

ref

$1.02(0.97-1.08)$

$0.69(0.64-0.75)$

$0.85(0.77-0.95)$

$1.88(1.56-2.28)$

$0.49(0.32-0.73)$

$1.46(0.91-2.37)$

$3.86(1.68-8.86)$

$0.65(0.53-0.81)$

$1.42(1.09-1.84)$

$2.90(2.01-4.17)$

Fig. 3 Association between vitamin C monotherapy and hospital mortality compared with in combination with corticosteroids and/or thiamine. The numbers and percentages of patients who died according to each drug combination are shown. The odds ratios (ORs) and $95 \%$ confidence intervals (Cls) are calculated in the propensity score (PS)-matched treatment and control groups

\section{Discussion}

This nationwide cohort study with PS analysis found a significantly lower hospital mortality among patients with sepsis treated with IV vitamin $C$ of $\geq 5$ days. These findings were consistent for patients with older age, multiple comorbidities, pneumonia, septic shock, and mechanical ventilation. In addition, vitamin $C$ alone or in combination with thiamine, but not with corticosteroids, was associated with improved mortality.

The hospital mortality rates of patients with sepsis and septic shock were $17.1 \%$ and $28.7 \%$, respectively. These are in line with the $10 \%$ mortality rate in the Sepsis-3 criteria [1] and the $24.8-28.2 \%$ mortality rates of septic shock in a recent study using a US healthcare database [24]. To date, several randomized trials have evaluated the efficacy of vitamin $\mathrm{C}$ as an adjunctive therapy for sepsis, but the results have been conflicting [7-10]. For the primary outcomes, these trials used SOFA scores and ventilator- and vasopressor-free days instead of mortality owing to lack of power. In addition, a considerable proportion of patients received corticosteroids, further diluting the effect size. The main strength of this study is the use of the largest national data in existence to assess the association between vitamin $\mathrm{C}$ and mortality in sepsis patients while controlling for the use of corticosteroids. Another strength of the study was that there were no missing values in the database. The results revealed a decrease of both hospital and long-term mortality in patients who received vitamin $C$ even after PS matching. However, $0.9-1.6 \%$ differences in mortality may not be considered to be clinically important compared with a large effect size demonstrated in previous observational studies [6].

Sepsis could downregulate the cellular sodiumdependent vitamin $C$ transporters [25]. Thus, whether vitamin $C$ saturation in the plasma reflects comparable saturation of septic tissues is unclear. The present results demonstrated that patients who received vita$\min C$ for $\geq 5$ days had a significantly lower mortality. This finding may be important and clinically relevant, given that most previous studies, which failed to show survival benefit, limited the use of vitamin $C$ to 4 days. Despite high-dose administration of vitamin $C$, patients may experience hypovitaminosis $\mathrm{C}$ within several hours of discontinuation [26]. In the CITRIS-ALI trial, the survival curve of the vitamin $C$ group became parallel to that of the placebo group after cessation of treatment [7], suggesting that the favorable effects of vitamin C may decrease over time. The lack of consistent benefits in previous trials of vitamin $\mathrm{C}$ in sepsis may also be due to insufficient dosage. For instance, the ACTS trial, which showed negative results, evaluated patients who received only 1 dose of study drug [9]. Conversely, the CITRIS-ALI trial tested a higher dose of vitamin $\mathrm{C}(200 \mathrm{mg} / \mathrm{kg} /$ day $)$ and found a significant decrease in mortality among patients with sepsis and acute 
respiratory distress syndrome [7]. Interestingly, a recent meta-analysis revealed the associations between highdose ( $\geq 6$ to $<12 \mathrm{~g} /$ day) and very high-dose ( $\geq 12 \mathrm{~g} /$ day) vitamin $C$ and decreased mortality [13].

The outcomes in previous trials may have been influenced by patient heterogeneity. Moreover, there is a possibility that vitamin $C$ may also be beneficial in certain subgroups. In many cases, clinical trials of interventions for sepsis excluded very elderly ( $\geq 70$ years) patients with multiple comorbidities due to a high risk of death and low treatment response. However, these patients accounted for a large proportion (59\%) of the present population who showed lower mortalities when vitamin $C$ was administered. Notably, there was no mortality decrease with vitamin $C$ in patients with younger age ( $<70$ years) and less comorbidities, suggesting that treating specific subgroups may have resulted in improved overall mortality. The association between vitamin $\mathrm{C}$ and improved mortality was also greater for patients with pneumonia and mechanical ventilation. Vitamin $\mathrm{C}$ administration to peripheral blood monocytes from pneumonia patients decreased proinflammatory cytokines [27]. Furthermore, several studies have shown that vitamin $C$ decreased the duration of mechanical ventilation and mortality in patients with severe pneumonia [16, 28]. In the current study, vitamin $C$ was significantly associated with a lower hospital mortality in patients with septic shock, but not in patients with renal replacement therapy. A possible explanation for this may be vitamin $\mathrm{C}$ loss by filtration, dialysis, or non-reabsorption [29]. Long-term vitamin C might be useful, as the present data revealed that patients in this subgroup who received vitamin $C$ for $\geq 5$ days had a significantly lower mortality.

Previous trials did not evaluate the individual effects of vitamin $C$ and thiamine. In this study, vitamin $C$ was associated with increased mortality in sepsis patients in the presence of corticosteroids. In addition, corticosteroid use was associated with a higher risk of mortality after adjusting for confounding due to baseline severity. The increased risk of neuromuscular weakness from corticosteroids among relatively older patients in the present study might explain these findings [30]. Meanwhile, mortality was significantly lower for patients with septic shock and mechanical ventilation who received short-term corticosteroids. These are in line with recent guidelines that advocate for the use of corticosteroids in septic shock [31, 32]. However, it may be noted that several meta-analyses showed that vitamin $\mathrm{C}$ monotherapy was more beneficial than when administered in combination with corticosteroids $[13,33]$. Interestingly, the combination of vitamin $C$ and thiamine was associated with a further lower mortality in certain subgroups, while other studies have not shown this finding [13,33]. Additional studies are required to determine potential optimized drug combinations.

This study has several limitations. First, its non-randomized design precludes any inference of causality on association between vitamin $\mathrm{C}$ use and mortality. Moreover, a retrospective design is vulnerable to unmeasured confounders, although the groups were balanced with respect to measured confounders using PS analysis. Second, vital signs and laboratory data were lacking in the database, but diagnoses, prescriptions, and procedures were used as surrogates of disease severity. Third, sepsis definitions which are based on administrative codes can be affected by coding practices unrelated to the physiologic data [34, 35]. Moreover, sepsis was defined as infection and organ dysfunction within the same admission, but a time difference may exist. Fourth, analytical methods to address immortal time bias may be insufficient due to inability to temporally associate diagnoses with treatments. Data on timing of ICU admission, vasopressor initiation, or vitamin $\mathrm{C}$ administration were unavailable due to lack of time stamps. Thus, whether early or delayed vitamin $C$ treatment is associated with outcomes among sepsis patients could not be assessed in the present study. Fifth, total vasopressor days, ventilator days, and ICU and hospital lengths of stay were calculated because the sequence of events within each admission was not available. In addition, the hospital costs were all-cause estimates and not the attributable costs of sepsis. These might explain the findings that vitamin $C$ was associated with a lower hospital mortality but not with improvements in secondary outcomes. Sixth, this study did not assess the outcome of different doses of vitamin C. Seventh, vitamin C levels were not available, and patients in this study were not screened based on their vitamin $C$ status.

\section{Conclusions}

In this nationwide cohort study of patients with sepsis, intravenous vitamin $\mathrm{C}$ is associated with a lower hospital mortality. However, the present results should be interpreted with caution because the difference of mortality was extremely small. The finding that vitamin $\mathrm{C}$ of $\geq 5$ days was associated with an even greater decrease in mortality is consistent with previous meta-analysis suggesting that there may be a dose-response relationship between vitamin $C$ and mortality [13]. Additional randomized trials evaluating very high-dose, long-term vitamin $C$ for sepsis are warranted. Further studies are also needed to evaluate the efficacy of vitamin $C$ in specific subpopulations with sepsis that may benefit from treatment, using optimized combination with corticosteroids and/or thiamine. 


\section{Abbreviations}

CCl: Charlson Comorbidity Index; Cl: Confidence interval; ICD: International Classification of Diseases; ICU: Intensive care unit; IV: Intravenous; NHIS: National Health Insurance Service; OR: Odds ratio; PS: Propensity score; SD: Standard deviation; SOFA: Sequential Organ Failure Assessment.

\section{Supplementary Information}

The online version contains supplementary material available at https://doi. org/10.1186/s13054-021-03872-3.

Additional file 1: Appendix 1. ICD-10 codes used for identification of infectious condition.

Additional file 2: Table S1. ICD-10-based classification of organ dysfunction. Table S2. Anatomical Therapeutic Chemical codes and charge codes for intravenous vitamin C, corticosteroids, and thiamine. Table S3. ICD-10 codes for different sites of infection. Table S4. Baseline patient characteristics in the unmatched cohort. Table S5. Primary and secondary outcomes according to ICU length of stay. Table S6. Secondary outcomes of patients who survived to hospital discharge. Table S7. Risk factors for hospital mortality. Table S8. Primary and secondary outcomes according to vitamin C duration. Table S9. Primary and secondary outcomes in patients who received vitamin C for $\geq 5$ days and in matched controls. Table S10. Primary and secondary outcomes according to ICU length of stay in patients who received vitamin $\mathrm{C}$ for $>5$ days and in matched controls. Table S11. Primary and secondary outcomes by subpopulations. Table S12. Primary and secondary outcomes by subpopulation in patients who received vitamin $C$ for $\geq 5$ days and in matched controls.

Additional file 3: Fig. S1. Survival from hospital admission to day 90 by subpopulations. Fig. S2. Survival from hospital admission to day 90 by sepsis subpopulations among patients who received vitamin C for $\geq 5$ days and matched controls. Fig. S3. Association between vitamin C monotherapy and hospital mortality compared with in combination with corticosteroids and/or thiamine in the sepsis subpopulations.

\section{Acknowledgements}

Not applicable.

\section{Authors' contributions}

WYK participated in the conception and design of study. SYJ and MTL participated in the data acquisition and data analysis. WYK, SYJ, and MSB participated in the data interpretation. WYK and SYJ participated in the draft of the manuscript. MTL and MSB helped to revise the manuscript for important intellectual content. All authors read and approved the final manuscript.

\section{Funding}

This research was supported by the National Research Foundation of Korea grant funded by the Korea government (Ministry of Science, ICT \& Future Planning) (2019R1G1A1099373), who paid the costs of using the Korean National Health Insurance Service database. The funders had no role in the design and conduct of the study; collection, management, analysis, and interpretation of the data; preparation, review, or approval of the manuscript; or decision to submit the manuscript for publication.

\section{Availability of data and materials}

All data generated or analyzed during this study are included in this published article and its supplementary information files.

\section{Declarations}

Ethics approval and consent to participate

The need for ethical approval was waived by the Institutional Review Board of Chung-Ang University owing to the de-identified patient data (1041078-202001-HR-012-01).

\section{Consent for publication}

Not applicable.

\section{Competing interests}

The authors declare that they have no competing interests.

\section{Author details}

${ }^{1}$ College of Pharmacy, Chung-Ang University, Seoul, Republic of Korea. ${ }^{2}$ Department of Global Innovative Drugs, The Graduate School of Chung-Ang University, Chung-Ang University, Seoul, Republic of Korea. ${ }^{3}$ Division of Pulmonary and Critical Care Medicine, Department of Internal Medicine, Chung-Ang University Hospital, Chung-Ang University College of Medicine, Seoul, Republic of Korea.

Received: 3 November 2021 Accepted: 11 December 2021

Published online: 05 January 2022

\section{References}

1. Singer M, Deutschman CS, Seymour CW, Shankar-Hari M, Annane D, Bauer $M$, et al. The third international consensus definitions for sepsis and septic shock (Sepsis-3). JAMA. 2016;315:801-10.

2. Fleischmann C, Scherag A, Adhikari NK, Hartog CS, Tsaganos T, Schlattmann $P$, et al. Assessment of global incidence and mortality of hospitaltreated sepsis. Current estimates and limitations. Am J Respir Crit Care Med. 2016;193:259-72.

3. Marshall JC. Why have clinical trials in sepsis failed? Trends Mol Med. 2014:20:195-203.

4. Wilson JX. Mechanism of action of vitamin C in sepsis: ascorbate modulates redox signaling in endothelium. BioFactors. 2009;35:5-13.

5. Carr AC, Shaw GM, Fowler AA, Natarajan R. Ascorbate-dependent vasopressor synthesis: a rationale for vitamin $\mathrm{C}$ administration in severe sepsis and septic shock? Crit Care. 2015;19:418.

6. Marik PE, Khangoora V, Rivera R, Hooper MH, Catravas J. Hydrocortisone, vitamin C, and thiamine for the treatment of severe sepsis and septic shock: a retrospective before-after study. Chest. 2017;151:1229-38.

7. Fowler AA 3rd, Truwit JD, Hite RD, Morris PE, DeWilde C, Priday A, et al. Effect of vitamin $C$ infusion on organ failure and biomarkers of inflammation and vascular injury in patients with sepsis and severe acute respiratory failure: the CITRIS-ALI randomized clinical trial. JAMA. 2019:322:1261-70.

8. Fujii T, Luethi N, Young PJ, Frei DR, Eastwood GM, French CJ, et al. Effect of vitamin C, hydrocortisone, and thiamine vs hydrocortisone alone on time alive and free of vasopressor support among patients with septic shock: the VITAMINS randomized clinical trial. JAMA. 2020:323:423-31.

9. Moskowitz A, Huang DT, Hou PC, Gong J, Doshi PB, Grossestreuer AV, et al. Effect of ascorbic acid, corticosteroids, and thiamine on organ injury in septic shock: the ACTS randomized clinical trial. JAMA. 2020;324:642-50.

10. Sevransky JE, Rothman RE, Hager DN, Bernard GR, Brown SM, Buchman TG, et al. Effect of vitamin C, thiamine, and hydrocortisone on ventilatorand vasopressor-free days in patients with sepsis: the VICTAS randomized clinical trial. JAMA. 2021;325:742-50.

11. Venkatesh B, Finfer S, Cohen J, Rajbhandari D, Arabi Y, Bellomo R, et al. Adjunctive glucocorticoid therapy in patients with septic shock. N Engl J Med. 2018;378:797-808.

12. Annane D, Renault A, Brun-Buisson C, Megarbane B, Quenot JP, Siami S, et al. Hydrocortisone plus fludrocortisone for adults with septic shock. N Engl J Med. 2018;378:809-18.

13. Fujii T, Salanti G, Belletti A, Bellomo R, Carr A, Furukawa TA, et al. Effect of adjunctive vitamin $C$, glucocorticoids, and vitamin B1 on longer-term mortality in adults with sepsis or septic shock: a systematic review and a component network meta-analysis. Intensive Care Med. 2021. https://doi. org/10.1007/s00134-021-06558-0.

14. National Health Insurance Service. Population coverage (2018). https:// www.nhis.or.kr/english/wbheaa02400m01.do. Accessed 3 Nov 2021.

15. Vail EA, Gershengorn HB, Wunsch H, Walkey AJ. Attention to immortal time bias in critical care research. Am J Respir Crit Care Med. 2021;203:1222-9.

16. Kim WY, Jo EJ, Eom JS, Mok J, Kim MH, Kim KU, et al. Combined vitamin C, hydrocortisone, and thiamine therapy for patients with severe pneumonia who were admitted to the intensive care unit: propensity score-based analysis of a before-after cohort study. J Crit Care. 2018;47:211-8. 
17. Scholz SS, Borgstedt R, Ebeling N, Menzel LC, Jansen G, Rehberg S. Mortality in septic patients treated with vitamin C: a systematic meta-analysis. Crit Care. 2021;25:17.

18. Charlson ME, Pompei P, Ales KL, MacKenzie CR. A new method of classifying prognostic comorbidity in longitudinal studies: development and validation. J Chronic Dis. 1987;40:373-83.

19. Rosenbaum PR, Dubin DB. The central role of the propensity score in observational studies for causal effects. Biometrika. 1983;70:41-55.

20. Seung KB, Park DW, Kim YH, Lee SW, Lee CW, Hong MK, et al. Stents versus coronary-artery bypass grafting for left main coronary artery disease. $\mathrm{N}$ Engl J Med. 2008;358:1781-92.

21. Carr AC, Spencer E, Dixon L, Chambers ST. Patients with community acquired pneumonia exhibit depleted vitamin C status and elevated oxidative stress. Nutrients. 2020;12:1318.

22. Hickling DR, Nitti VW. Management of recurrent urinary tract infections in healthy adult women. Rev Urol. 2013;15:41-8.

23. El-Gebaly E, Essam T, Hashem S, El-Baky RA. Effect of levofloxacin and vitamin $C$ on bacterial adherence and preformed biofilm on urethral catheter surfaces. J Microb Biochem Technol. 2012;4:131-6.

24. Vail EA, Wunsch H, Pinto R, Bosch NA, Walkey AJ, Lindenauer PK, et al. Use of hydrocortisone, ascorbic acid, and thiamine in adults with septic shock. Am J Respir Crit Care Med. 2020;202:1531-9.

25. Subramanian VS, Sabui S, Moradi H, Marchant JS, Said HM. Inhibition of intestinal ascorbic acid uptake by lipopolysaccharide is mediated via transcriptional mechanisms. Biochim Biophys Acta Biomembr. 2018;1860:556-65.

26. de Grooth HJ, Manubulu-Choo WP, Zandvliet AS, Spoelstra-de Man AME, Girbes AR, Swart EL, et al. Vitamin C pharmacokinetics in critically ill patients: a randomized trial of four IV regimens. Chest. 2018;153:1368-77.

27. Chen $Y$, Luo G, Yuan J, Wang Y, Yang X, Wang $X$, et al. Vitamin C mitigates oxidative stress and tumor necrosis factor-alpha in severe communityacquired pneumonia and LPS-induced macrophages. Mediators Inflamm. 2014;2014:426740.

28. Hemila H, Chalker E. Vitamin C may reduce the duration of mechanical ventilation in critically ill patients: a meta-regression analysis. J Intensive Care. 2020;8:15

29. Kamel AY, Dave NJ, Zhao VM, Griffith DP, Connor MJ Jr, Ziegler TR. Micronutrient alterations during continuous renal replacement therapy in critically ill adults: a retrospective study. Nutr Clin Pract. 2018;33:439-46.

30. Rochwerg B, Oczkowski SJ, Siemieniuk RAC, Agoritsas T, Belley-Cote E,

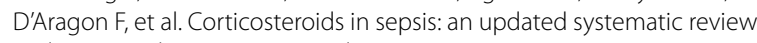
and meta-analysis. Crit Care Med. 2018:46:1411-20.

31. Lamontagne F, Rochwerg B, Lytvyn L, Guyatt GH, Moller MH, Annane D, et al. Corticosteroid therapy for sepsis: a clinical practice guideline. BMJ. 2018;362:k3284

32. Evans L, Rhodes A, Alhazzani W, Antonelli M, Coopersmith CM, French C, et al. Surviving sepsis campaign: international guidelines for management of sepsis and septic shock 2021. Intensive Care Med. 2021:47:1181-247.

33. Fong KM, Au SY, Ng GWY. Steroid, ascorbic acid, and thiamine in adults with sepsis and septic shock: a systematic review and component network meta-analysis. Sci Rep. 2021;11:15777.

34. Rhee C, Dantes R, Epstein L, Murphy DJ, Seymour CW, Iwashyna TJ, et al. Incidence and trends of sepsis in US hospitals using clinical vs claims data, 2009-2014. JAMA. 2017:318:1241-9.

35. Johnson AEW, Aboab J, Raffa JD, Pollard TJ, Deliberato RO, Celi LA, et al. A comparative analysis of sepsis identification methods in an electronic database. Crit Care Med. 2018;46:494-9.

\section{Publisher's Note}

Springer Nature remains neutral with regard to jurisdictional claims in published maps and institutional affiliations.
Ready to submit your research? Choose BMC and benefit from:

- fast, convenient online submission

- thorough peer review by experienced researchers in your field

- rapid publication on acceptance

- support for research data, including large and complex data types

- gold Open Access which fosters wider collaboration and increased citations

- maximum visibility for your research: over $100 \mathrm{M}$ website views per year

At BMC, research is always in progress.

Learn more biomedcentral.com/submissions 